\title{
Integration of robust and tolerance design in early stages of the product development process
}

\author{
Stefan Goetz ${ }^{1}$ (1) $\cdot$ Benjamin Schleich ${ }^{1} \cdot$ Sandro Wartzack $^{1}$
}

Received: 24 September 2018 / Revised: 5 December 2019 / Accepted: 9 December 2019 / Published online: 8 February 2020

(c) The Author(s) 2020

\begin{abstract}
Due to the tightening of requirements and the increasing complexity of products, robust design becomes more and more important in the context of a straightforward product development. Although various robust design methods have been evolved to support the design of products that are less sensitive to variations, these methods are not sufficiently integrated into early stages of the product development process. This is mainly due to the lack of concrete product data necessary for robustness evaluation and tolerance specification. For this reason, it is still unclear to product development engineers when and how to apply existing approaches for enhancing the robustness of products. Therefore, this paper describes a holistic methodology that supports the designer in developing a robust product layout including an initial, validated tolerance specification based on the functional requirements. The proposed framework focuses on the close linkage of robust design activities with product data along the product development process and demonstrates the resulting benefits of an early consideration of variations and tolerances. In addition to the proper choice of robust product concepts and their corresponding spatial arrangement in the product structure, the approach allows a conceptual tolerance specification and the subsequent CADbased analysis based on the preliminary design. Thus, the proposed approach aims to reduce iterations in the conventional tolerancing. To demonstrate the process, the methodology is applied to an electric window regulator.
\end{abstract}

Keywords Robust design · Tolerance design · Early design stages · Concept and preliminary design

\section{Introduction}

The increasing complexity of products in combination with the shortening of product lifecycles dictates a stringent application of quality management strategies. While popular practices, such as Total Quality Management or Six Sigma, mainly focus the adaption of the production process, the potential of a quality-driven product development, usually called robust design, often remains unused (Eifler and Howard 2018). Although the industry is aware of the importance of robust design, it is rarely used in practice (Gremyr and Hasenkamp 2011). This is partly due to the fact that the vast number of different approaches each considers only single aspects of the robust design paradigm. However, for a successful integration into the product development process, it is advisable to take into account many different aspects

Stefan Goetz

goetz@mkf.fau.de

1 University Erlangen-Nürnberg, Engineering Design, Martensstrasse 9, 91058 Erlangen, Germany
(Eifler and Howard 2018). This fundamental premise can be attributed to Taguchi, who is a pioneer in the field of robust design and splits the process into the three phases, systems/ concept design, parameter design and tolerance design as it is shown in Fig. 1.

This emphasizes that robust design covers the entire product development process, while tolerance design is just one factor contributing to the functionality of a product. In this regard, various publications stress the importance of the product concept in terms of the robustness of the final product (Jugulum and Frey 2007; Ebro et al. 2012). Although there are controversial studies on the influence of the product concept on the final product quality (Ulrich and Pearson 1993; Hsu and Woon 1998), the relevance of the concept in the context of product development is undisputed (Pahl et al. 2007; Reich 2008; Ullmann 2009). This is in line with the common principle that especially design changes in the early stages have a high cost effect and can, thus, significantly contribute to the economic development of products. In combination with the close link of the concept and parameter design with the product development activities, 


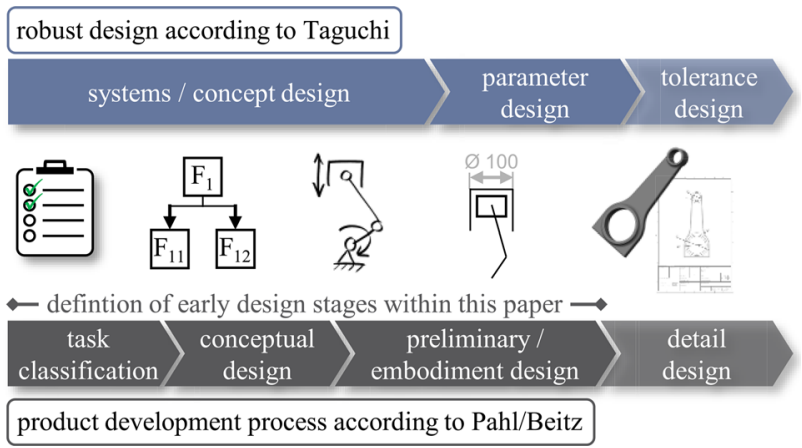

Fig. 1 Timing of robust design activities (Taguchi et al. 1990) in the product development process (Pahl et al. 2007)

this implies that variations should be taken into account as early as possible in the design process. However, in analogy to problems in the early product design (Hsu and Woon 1998; Lough et al. 2009), the lack of geometry information, which usually forms the basis for the conventional tolerancing process, poses a major challenge in the early stages. Although the available approaches enabling the consideration of robust design aspects even prior to the final geometry definition partly address this challenge, a continuous robust design methodology is still missing. Therefore, this paper proposes an approach that allows the designer to integrate robust and tolerance design in the early stages of the product development process starting from the task classification up to the preliminary design stage. The fundamental underlying idea described in this contribution is to provide a method by a close linkage of the product development and the robust and tolerance design activities. It, thus, contributes to the intensive combination of both domains, which enables the frontloading of robust design and tolerancing activities.

The paper is structured as follows. First of all, related work regarding the consideration of variations during the different stages of the product development process is presented. Subsequently, Sect. 3 introduces the integrated Robust and tolerance design approach and describes the individual steps for their thorough application. In Sect. 4, the proposed approach is exemplarily applied to an electric window regulator and subsequently discussed in Sect. 5 . Finally, in Sect. 6 a summary and an outlook are given.

\section{Related work and state of the art}

While the conventional tolerance design can be regarded as a single phase at the end of the design process (Polini 2016), the consideration of variations in early stages is rather to be understood as part of the whole product development

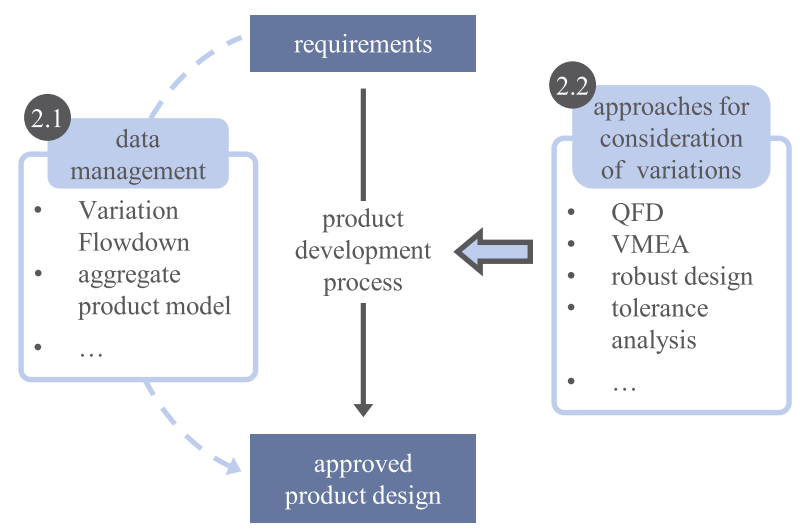

Fig. 2 Classification of approaches that allow designers to consider variations during the product development process

process. Although approaches for the integration of tolerance specifications into the product development [such as (Islam 2009)] already exist, the development of approaches for the consideration of variations in early phases does not consider the workflow of the design process. Thus, a clear assignment of these approaches to the stages of the product development process is often difficult (Göhler et al. 2017) or the practical application takes place later than actually possible in the development process due to a lack of expert knowledge or abstraction ability. This is in line with the findings that, besides the development of individual useful approaches, their integration into the process is a key aspect (Eifler et al. 2013). Accordingly, the state of the art is divided into two sections corresponding to the categories 'data management' (Sect. 2.1) representing the link to the design process and the 'approaches for consideration of variation' (Sect. 2.2), see Fig. 2. This corresponds to the two fundamentally different concepts for taking into account variations during the transformation of the requirements to the final product design. In the sense of a top-down-oriented product development, data management approaches offer the possibility to represent the product data in a structured way and to progressively expand it. By visualizing the relationships between the different product data, a comprehensive data management can implicitly support the tolerance driven product development. For example, a link between functional requirements and design parameters helps to ensure a function-oriented tolerance design. On the other hand, there are tools and methods that locally guide the designer in individual activities to take robust design aspects into account in the product development process. Thus, these approaches support the specific adaption of the product and tolerance design to develop products that meet the functional requirements while taking variations into account. 


\subsection{Data management}

Data models are mainly used to ensure the traceability and consistency of the product development process. The benefit of this becomes clear in the final tolerance design, where the requirements defined at the beginning of product development form the basis for a function-oriented tolerancing (Srinivasan et al. 1996). Thus, the basic idea of the proposed data models is to break down the functional requirements to specific design parameters (Suh 1998; Mathieu and Marguet 2001). In this context, Thornton developed the variation flowdown that transforms the product characteristics into part and process parameters (Thornton 2004). The key characteristics defined in this framework and meanwhile widely used describe parameters that have a particular impact on the variation of the products' quality (Thornton 1999). The extension of these top-down structures of relevant characteristics allows the mapping of interactions between them. Translating this structure into matrices enables quantification and thus fosters the breakdown to nominal dimensions and tolerances (Johannesson and Söderberg 2000). Dantan et al. (2003) further enhances this mainly function-oriented structures with assembly information, which results in an object-oriented model that comprises data from both disciplines, namely product and tolerance design.

Further similar data models focus on the management of the progressive concretization of product and tolerance information associated with the product development process. For example, the aggregate product model according to Bradley and Maropoulosa allows to break down the entire product into individual components, which represent a combination of features with the corresponding relevant tolerance information at the lowest level (Bradley and Maropoulosa 1998). A similar top-down model approach is based on artifacts, which are successively supplemented with information such as requirements, functions, geometry and tolerances as well as their interrelations (Roy et al. 2001). The abstract tolerance information derived from the functional requirements (e.g., part a parallel to part b) supports the tolerance design at the end of the product development. This improved communication of the design intentions enables an efficient tolerance design, especially for complex products (Bjarklev et al. 2018).

In summary, the numerous data management approaches allow a proper linking between functional requirements and the final product design including relevant concept information. However, the approaches are formulated in an abstract manner or require geometric information for the practical application. Therefore, it is often difficult for the designer to transfer this data management information to the tolerance-driven product development process.

\subsection{Approaches for the consideration of variations in the product development process}

To support the designer in the development of robust products, numerous tools and methods applicable in the different stages of the product development process have been evolved. Due to the close linkage, some of them are similar or analogous to established design methods. In accordance with the progressing design process, the presented approaches are arranged starting from an abstract level up to the concrete tolerance design.

At the beginning of the product development process, the robust design activities are adopted from the striving to map the relationship between design, product properties and the resulting fulfillment of requirements, for example with the aid of quality management. In a first step, the quality function deployment (QFD) can be used to translate the customer's needs into concrete functional requirements (Sullivan 1986). Similar to the domain-based approach according to Suh (1990), it is, thus, possible to determine how the respective requirements are to be addressed throughout the entire development process (Göhler et al. 2016). Thus, the idea of modern custom-oriented design methods, such as the Property-Driven Development (Weber et al. 2003), is transferred to the tolerance domain. However, in the interest of an efficient product development, it is important to limit oneself to the significant factors analogous to the key characteristics. But this distinction is often difficult, especially at the beginning, as the significance of the factors can usually only be assessed subjectively. Therefore, based on the failure mode and effect analysis (FMEA), the variation mode and effect analysis (VMEA) was developed and quantifies how likely it is that variation of individual factors influences the requirements (Chakhunashvili et al. 2004). This method can also be used in late development phases to quantify the interactions between influencing factors and the product characteristics in case of unknown transfer functions (Göhler et al. 2017). Although the VMEA is similar to the established risk evaluation in change management (Clarkson et al. 2004), the focus here is primarily on small variations in the context of the consideration of tolerances. Thus, it supports the creation of the variation management framework (VMF), in which the relationships between customer's needs, functional requirements, design parameters and process variables are quantitatively represented in a diagram with four corresponding quadrants (Howard et al. 2017).

Once again, this approach emphasizes the importance of a continuous consideration of variation during the design process. For this reason, there are a few robust design methods that can already be applied in the conceptual design and hence do not necessarily require detailed geometry information. Since the robust concept design has a greater effect on the robustness of final products than the parameter design 
(see Fig. 1), these methods are particularly important and should be applied prior to the classical robust design activities (Andersson 1997; Ebro et al. 2012). Thus, the formulas underlying the physical effects can be used for evaluating the robustness of principle solutions and thereby support their appropriate selection (Mathias et al. 2011). However, in the field of mechanical engineering, the required functional relationships often only emerge through the product design, e.g., during the development of mechanisms. For this reason, several design principles have been established to keep the influence of variation in design parameters on the functional performance to a minimum. Thus, the verification of the resulting degree of freedom of principle designs, which corresponds to the kinematic design, prevents the development of over-constrained systems (Myszka 2012). Ebro et al. (2012) adopts this basic idea at the interface level and thereby the design clarity procedure prevents unintended couplings within the contact area such as double fit. The axiomatic design has a similar claim to reduce interaction in the system and to keep functional requirements as well as design parameters independent of each other (Suh 1990). According to this, an extensive uncoupling between these two factors is aimed in the sense of a robust product (Söderberg and Lindkvist 1999). However, sometimes coupling is inevitable and even may be useful, for example, in the context of function integration. Therefore, the Contradiction Index was developed, which further distinguishes between positive and negative coupling (Göhler and Howard 2015). Accordingly, systems in which the variation of a parameter has a positive influence on the respective coupled functions are to be preferred to systems with opposing functional relationships. For further approaches in this context, please refer to additional literature, such as Andersson (1997), Matthiassen (1997) and Ebro and Howard (2016). In the context of the proposed robust concept development, the multitude of these robust design principles is the basis for the proper application of approaches for concept selection, which are extensively discussed in Kremer (2008). For instance, the principles can be used as criteria in the selection of concepts according to Pugh (1981) or in other design decision-making approaches.

The concept selection allows the creation of first drafts, which form the basis for the traditional robust design (parameter design acc. to Taguchi) including design of experiments (DOE), statistics and optimization (Ford 1996). In the VMF, this stage includes the linkage of the function and the design parameters via a transfer function (Eifler et al. 2013) that represents the sensitivity. Besides the parameter optimization of mechanisms, a suitable locating scheme should be developed since it has a significant impact on the robustness of the final product (Söderberg et al. 2016). Furthermore, similar to the tolerance design, parameter values and tolerances are optimized to minimize the variation of the functional performance
(Taguchi et al. 2005). For the robustness evaluation carried out in this context, numerous different variables exist and are comprehensively summarized by Göhler et al. (2016). However, since detailed information on the geometric relationships is required for this kind of comprehensive analysis, this robust design activities are primarily assigned to the beginning of the detail design stage. Thus, in the robust design process in early stages, there is a significant gap between the early concept design with only abstract information and the detail design stage.

So, the following robust design activities are already part of the tolerance design, which is rather assigned to detail design stages according to the definition in Fig. 1. However, since some aspects of the tolerance design serve as the basis for the methodology described in Sect. 3, this step is briefly summarized for reasons of completeness. It basically consists of tolerance specification, representation and analysis. The tolerance specification is usually done, when the product geometry is already finally defined. Since this process relies on the knowledge and experience of an engineer, it is often regarded as a challenging task (Colosimo and Senin 2011). As the comprehensive standards (e.g., ASME Y14.5, ISO 14405) do not address this issue, many generative specification methods supporting the designer have been developed. This allows a partially automation of the tolerance specification with the help of a rule-based translation of specific user-defined assembly and functional requirements (Armillotta 2013). In a similar way, the CLIC tolerancing method enables the generation of a comprehensive specification (Anselmetti 2006).

For the application of these methods, the design intention must be clearly known in the context of a functionoriented tolerancing. In addition to the data models (Qin et al. 2017), two different approaches have been developed for the representation of the tolerances assigned (Dantan and Qureshi 2009). With regard to the tolerance analysis, spatial math models allow the representation of the tolerance zone resulting from the superposition of the assigned tolerances (Ameta et al. 2011). Alternatively, tolerance models are often described using the formula relationships between the varying parameters and the functional key characteristics. These relationships, e.g., depicted via matrices (Polini 2012) or vectors (Colosimo and Senin 2011), are the basis for the subsequent tolerance analysis. Moreover, there are numerous commercial tools such as $\mathrm{RD} \mathrm{T}^{\circledR}, 3 \mathrm{DCS}{ }^{\circledR}, \mathrm{VSA}^{\circledR}$ or Enventive ${ }^{\circledR}$ which are often used in industry. These approaches and tools allow the evaluation of the distribution of the key characteristic as well as its sensitivity to the variation of parameters usually represented by contribution in percentages (Shah et al. 2007). This closes the loop to the robust design, where these results serve as a basis for the tolerance and, if applicable, parameter optimization. 


\subsection{Discussion of the state of the art}

It can be concluded that there are numerous single methods and tools that enable to take variations into account along the product development process. However, the practical application in the design engineering environment is complex or requires detailed product information which are lacking in the early design stages. This discrepancy mainly occurs due to the fact that the development of approaches taking variation into account has mostly been detached from the product development process, making their integration into the practical design process difficult. Although this paper has already been trying to structure the numerous approaches from the different disciplines according to the product development process, a clear order for the stringent implementation of the robust and tolerance design is still missing (Göhler et al. 2017). This poses the design engineer with the challenge that an adaptation of the product development process or expert knowledge as well as a good abstraction ability is necessary for the continuous application of the methods. All these points constitute a hurdle for a product development accompanying consideration of variations.

Furthermore, there is a large discrepancy between the benefits and applicability of robust design methods, especially in early product development phases, see Fig. 3. After the geometry of a product has been defined, there are numerous robust and tolerance design approaches that are described in detail and partly implemented in commercial tools. However, in particular the consideration of variations prior to the definition of the geometry has great potential in the sense of an efficient product development (Andersson 1997). Nonetheless, there is a lack of concrete tools (Hasenkamp et al. 2009) that enable a meaningful integration of robust design aspects into the product development process. This finally leads to the fact that the potential of the robust design remains widely unused in industry (Eifler et al. 2013).

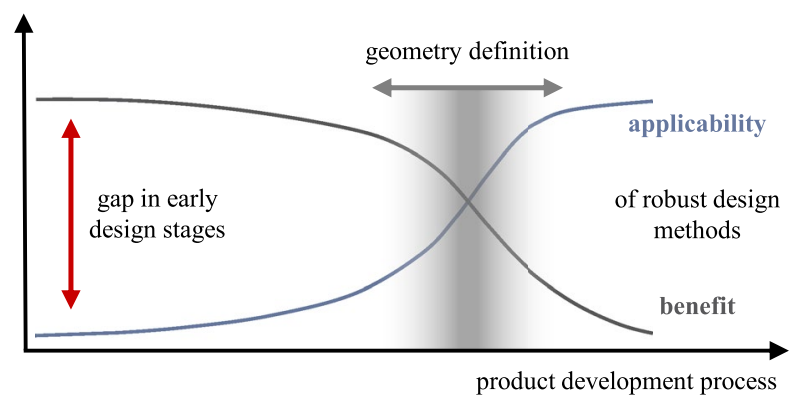

Fig. 3 Change of applicability and benefit of robust design methods during the development process (self-generated qualitative curves summarizing the findings of the literature review in this context)

\section{Integrated robust design and tolerancing process}

To overcome the highlighted shortcoming, this paper presents a holistic methodology that addresses the open questions about applicability and timing of robust design activities within the early stages of the development process. To meet the needs for a reliable and useful methodology, the structured development procedure is based on the four stages of the design research methodology (DRM) (Blessing and Chakrabarti 2009). Apart from the extensive literature review summarized in Sect. 2, the dialog with experts from automotive industry, among others, has shown that the current robust design in practice is rather an iterative process than a systematic approach to enhance the robustness of products in the sense of the first time right philosophy. Taking the different aspects (product, social and institutional) of the PSI framework (Reich and Subrahmanian 2015) into account finally helped to clarify that this is mainly due to the lacking knowledge accessibility in the early design stages. In combination with the discrepancy between the two domain perspectives of the design engineer and the tolerance expert, this contributes to expensive iterations at the end of the design process. This raises the following research question: how can a design engineer be enabled to consider aspects of robust design and tolerancing during the early stages of the product development to have a robust product design prior to the final detail design by a tolerance expert? The aim is to shift some of the competences of a tolerance expert to the product developer, so that the limited tolerancing resources can be used efficiently for the tolerance assurance. According to the DRM, above all, the simple data and knowledge transfer between both domains as well as the realization of a high usability of the method in the environment known to the designer were determined as essential success factors.

Since product development processes form the framework for the work of a design engineer, the essential recurring milestones (see upper path in Fig. 4) are the basis for the development of early applicable robust design or tolerancing methods, in which each is assigned to a specific product development stage. During their development, attention was paid to using methods and software which are already familiar to the design engineer, so that the hurdle in applying the integrated approach is kept to a minimum. Furthermore, the challenge of missing concrete information in early design stages is primarily met using implicit design and tolerance knowledge from the respective stages. Besides this close linkage of the individual method modules with the product development process, they were clustered in such a way that the output 


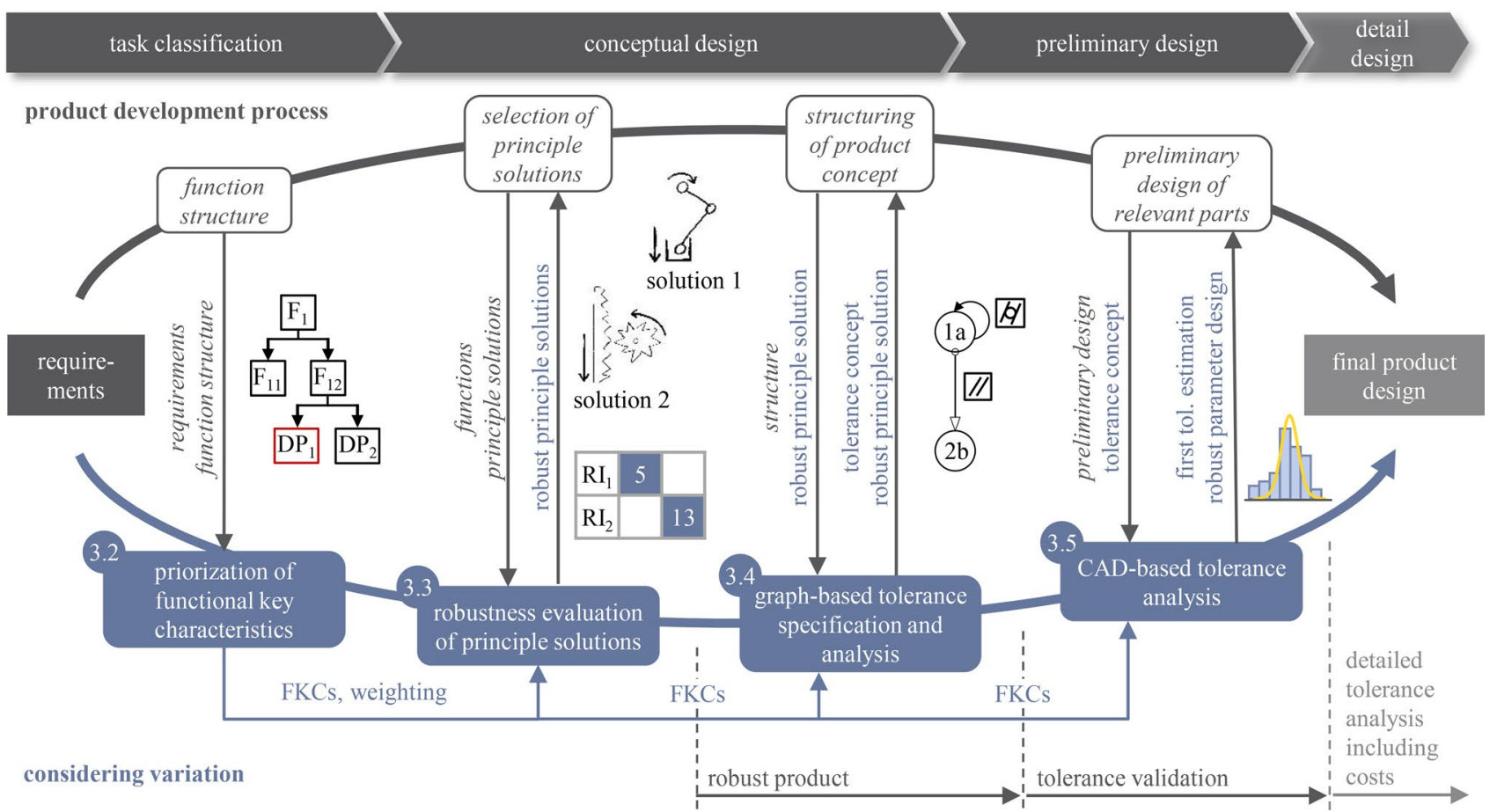

Fig. 4 Integration of robust and tolerance design activities in the first 3 stages of the product development process with corresponding information flow (gray highlighted italics is assigned to the product development, blue text is assigned to the proposed approach) (color figure online)

of the respective method serves as input for the following design step or for the application of the subsequent method for consideration of variation, see Fig. 4. Since the basic procedure of the individual methods [namely 'prioritization of FKCs and robustness evaluation of principle solutions' (Goetz et al. 2019), 'graph-based tolerancing' (Goetz et al. 2018a) and 'CAD-based tolerance analysis' (Goetz et al. 2018b)] has already been comprehensively described, the focus of this contribution is primarily on the presentation of the entire methodology and its continuous application to a product to demonstrate the general usability of the approach when thoroughly linking it to the product development process. In combination with the methods already described in the previous contributions, the proposed approach covers the early stages of the product development process from task classification to preliminary design and thereby creates a link to the detail design, where numerous established tools for the final product verification already exist.

\subsection{General framework}

Starting with the requirements from the product specification and the function structure, the integrated approach allows the identification of relevant functions and the associated functional key characteristics (FKC). As comprehensively described in the literature (Thornton 2004) and as can be seen in Fig. 4, the definition of key characteristics plays an important role during the entire tolerancing process. However, since the proposed approach focuses on the functionoriented tolerancing, the initial definition of key characteristics according to Thornton (2004) was adapted considering only functional relevant aspects. Moreover, since the product specification includes a comprehensive set of requirements, the FKCs are not only limited to customer interests but also respect internal aspects. Finally, FKCs are defined as product characteristics that are both sensitive to variation and at the same time elementary for meeting the requirements. Using a combination of a customized QFD and a newly developed method based on the basic VMEA approach, these identified elements are prioritized so that it becomes clear which functions and characteristics are particularly important for the further development, see Sect. 3.2.

As the design process progresses, several principle solutions are developed for each functional requirement. For the suitable selection of alternative solutions or sub-concepts (e.g., toggle lever, gear rack), the product development process includes an economic and technical evaluation. Since this selection in particular has a strong influence on the products robustness (Jugulum and Frey 2007), it is advisable to include the robust design aspect in the decision-making process. Therefore, the method described in Sect. 3.3 offers a newly developed robustness index allowing a robustness evaluation of concepts. A combination of established robust 
design principles, such as those exemplarily explained in the second paragraph of Sect. 2.2, provides the basis for this evaluation. In the context of the design process, the resulting index serves as an additional factor in a technical-economic evaluation and thus integrates the aspect of robust design into the selection of principle solutions.

Subsequently, the individual selected principle solutions are then modularized and combined into a rough product structure. Since this structure affects the robustness of the product (Goetz et al. 2018a), it is analyzed with the help of tolerance graphs so that the spatial arrangement of the solutions can be improved iteratively, see Sect. 3.4. Therefore, the minimum of geometric information, which is already predefined by the chosen principle solutions, is mapped in a graph and assigned with essential tolerances. This essential concretization step is supported by semantic knowledge about design and tolerance, which is already implicitly given by the spatial arrangement of principle solutions. By reducing the resulting tolerance graph according to the FKCs identified in the first step of the proposed methodology, the robustness of different concept structures is evaluated. In addition to a qualitative tolerance concept, a robust product concept with the corresponding spatial arrangement of the essential components is, thus, available at the end of the third step.

By adding fundamental dimensions, the previously chosen robust product structure is then transferred to a first preliminary design, such as a skeleton model. Referring to the early stages of the product development process, this represents the last milestone of the design process considered in this contribution. The models at this stage are usually used for the definition of interfaces in the concurrent product development or for an initial kinematic simulation. However, they can also be used for a quantitative tolerance analysis, so that the robust parameter design and, if applicable, the tolerance design can be carried out prior to the detail design. To improve the integration into the development process and the usability for the designer, the sampling-based analysis runs CAD internally on the basis of family tables, see Sect. 3.5. The open interface allows the results to be evaluated using established methods such as variance-based sensitivity analysis. This enables not only the creation of a robust product design but also an initial verification of elementary tolerances. In this way, the methodology for the early consideration of variation creates a meaningful link to the conventional process in the detail design stage where numerous established tools for tolerance analysis and synthesis exist.

As Fig. 4 already implies, the proposed procedure is, thus, applicable throughout the entire design process along the milestones from product specification to preliminary design and thus closes the gap of easy-to-use methods for the design engineer for taking into account variations in early stages of product development. Since this means that a first validation of elementary tolerances is already done in the preliminary design, expensive iterations in the final tolerance definition in the detail design stage can be reduced. The general possibility of integrating the individual methods within the product development process has already been demonstrated in preliminary work using various case studies, see Goetz et al. (2018a, b, 2019). In the following, the basic procedure of the required steps is described in the subsequent sections. Finally, its detailed application is shown in Sect. 4.

\subsection{Prioritization of functional key characteristics}

As Fig. 5 already implies, the process starts with a structure that represents the functions and subfunctions of a product. Since this initial structure usually only maps the topmost function levels, it is broken down similar to the variation flowdown (Thornton 2004) for a proper identification and consideration of critical variations. The lowest layer represents the functional key characteristics (FKC) that quantify the functional requirements. In this way, a parallelism requirement can be broken down to the two associated angles in three-dimensional space.

Even though the variation flowdown already presents a useful framework, the application of methods such as the House of Quality or FMEA is suggested for an effective break down process (Thornton 2004). In the proposed approach the established QFD method (Chan and Wu 2002) is adapted to support the design engineer in determining relevant characteristics and functions. In contrast to the wellknown first phase of the QFD process, which maps customer wishes and design requirements, here the functional requirements from the product specifications are linked to the functions or FKCs. Moreover, the correlation between both aspects is determined by specific questions. The strength of the correlation is subsequently used to prioritize, so that the subsequent process can be limited to relevant functions and FKCs. In this sense, less significant elements are highlighted in gray in the structure in the center of Fig. 5.

Moreover, beyond the procedure proposed by Thornton, the prioritization of these elements is of particular importance to be able to assess their significance with regard to

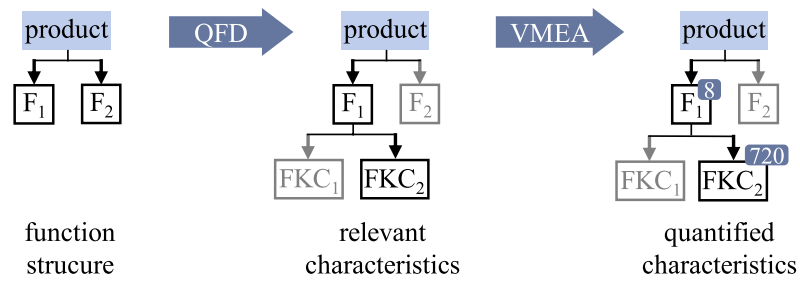

Fig. 5 Breakdown of function structure and prioritization of relevant characteristics 
the functional requirements of the product. For example, only one of the two descriptive angles could be relevant for the functional requirement 'parallelism'. However, since no quality loss functions or quantitative information about potential variation are available, classical methods are not applicable for this assessment. Instead, with respect to high usability in the design environment, a FMEA- or rather VMEA-oriented approach was developed to quantify the significance of individual FKCs across the different levels of the function structure with respect to the overall product. While the general procedure of the VMEA is maintained, the individual factors for the calculation of the customized variation risk priority number (VRPN) are modified, as comprehensively described in Goetz et al. (2019). Besides the evaluation of the significance of each function or FKC, it primarily respects possibly occurring variation and thus allows a quantified characterization of the importance of each element of the extended function structure. Finally, this weighting resulting from the adapted VMEA serves as an input for the subsequent robustness evaluation for the principle solutions.

\subsection{Robustness evaluation of principle solutions}

First, the functions and FKCs to be considered in the robustness evaluation are selected on the basis of the previously calculated VRPN values, taking into account the extent of the system under investigation and the available resources. Since these values are determined individually for each product, they are to be understood rather as a relative value, so that a generally valid threshold is not appropriate. However, the selected elements requiring further consideration are directly included in the robustness matrix with the corresponding VRPN values as weighting. This direct transfer from the previous step guarantees that the selection and weighting of relevant FKCs are independent of the principle solution, so that the alternative principle solutions coming from the product development process [e.g., in the form of a morphological matrix (Pahl et al. 2007)] can be evaluated neutrally in a common matrix.

Therefore, robust design criteria, which are combined within the matrix, are necessary, see Fig. 6. As comprehensively described in Goetz et al. (2019), established robustness criteria such as those described in Sect. 2.2 are used and individually weighted for this purpose. This meets the demand for a comprehensive robustness evaluation of the principle solutions. In concrete terms, it is evaluated to what extent the alternative principle solutions with regard to a certain function or FKC, for instance, realize the intended decoupling within the framework of the axiomatic design (Suh 1990). This means that solutions with a strong coupling between the design parameters are poorly evaluated in the matrix. As this case already shows, some of these criteria

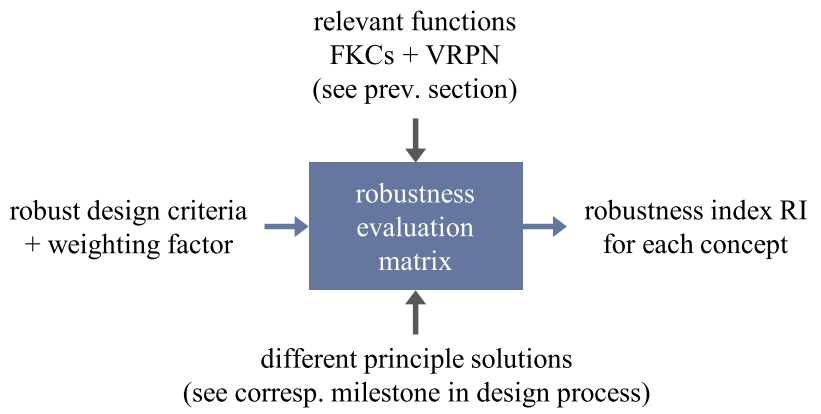

Fig. 6 Black box of the robustness evaluation of principle solutions with the corresponding input and output parameters

cannot be clearly assigned to a single FKC, but rather to a function or the whole product. Since these respective evaluations are respected with the corresponding weightings and VRPNs from Sect. 3.2, the robustness evaluation can be carried out on several levels (product, function and FKCs), so that the proposed matrix represents a unifying framework. All these aspects contribute to the usability and thus partly addresses the drawback of the numerous standalone approaches. The result of the procedure is a relative robustness index for the different principle solutions allowing the consideration of the robustness in the selection process in the context of product development. Thus, it contributes to the creation of robust concepts even before the geometry definition and forms the basis for further development.

\subsection{Graph-based tolerance specification and analysis of the product structure}

However, the spatial arrangement of the previously selected, robust partial solutions has not yet been specified and is, therefore, done in the progressing product development process. The resulting concept structure, exemplarily represented by a diagrammatic sketch (see Fig. 7), implies that the relevant geometric elements, especially in the contact areas, are already known prior to the actual geometric design of the product (Ballu et al. 2006). Although the functionality of a product can already be ensured at this stage (Ledoux and Teissandier 2013), the analysis is often challenging due to the missing visualization of the available information. For a proper mapping of this information, the proposed approach uses graphs, inspired by the established approaches widely used in the engineering environment (Zawiślak and Rysiński 2017) and mainly applied in the tolerance design (Ballu and Mathieu 1999; Giordano et al. 2007; Shen et al. 2008). This graphical visualization enables a qualitative tolerance specification of the concept and an initial analysis of the tolerance structure. As described in detail in Goetz et al. (2018a), the process starts with the product concept, see Fig. 7. 


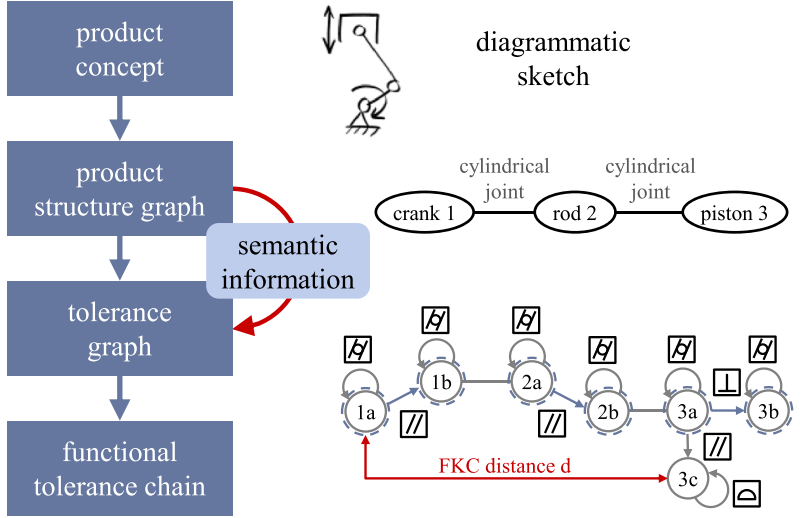

Fig. 7 Elementary process steps for the graph-based tolerance specification and analysis

Contrary to existing graph-based approaches, which are usually based on the final product geometry, this graph is generated step by step. In the first step, the diagrammatic sketch is transformed into the alternative product structure graph representing the structure of the relevant components and the corresponding interface relations. Subsequently, this graph at component level is extended to a graph at geometry element level. Since this can be challenging and requires implicit design knowledge, the process is partly automated with the help of semantic information, including geometry elements as well as tolerances according to the component and interface definition. Thus, a connecting rod contains a cylindrical bore at each of the two interfaces which are parallel to each other. In the tolerance graph in Fig. 7, it is represented by the two nodes 2 a and $2 \mathrm{~b}$ with the corresponding cylindricity tolerances for the bore surfaces. This fosters the generation of a tolerance graph comprising the relevant geometry elements and the comprehensive geometric tolerancing, that is feasible at this design stage. The graphical representation makes the arrangement as well as the relationships between the geometry elements clear, so that the resulting tolerance structure becomes comprehensible. Finally, the integration of the FKCs defined in Sect. 3.2 allows the identification of functional tolerance chains, so that it becomes clear which geometry elements and tolerances contribute to the FKC. By comparing the resulting tolerance chains of different concept structures, the spatial arrangement with comparatively high robustness can be identified taking into account the axiomatic design (Suh 1990) alongside an initial tolerance concept. This approach, thus, helps to validate the concept design and partly anticipates the tolerance design. Even though this cannot be clearly classified in the process according to Taguchi et al. (1990), it closes the gap between the abstract methods in the early

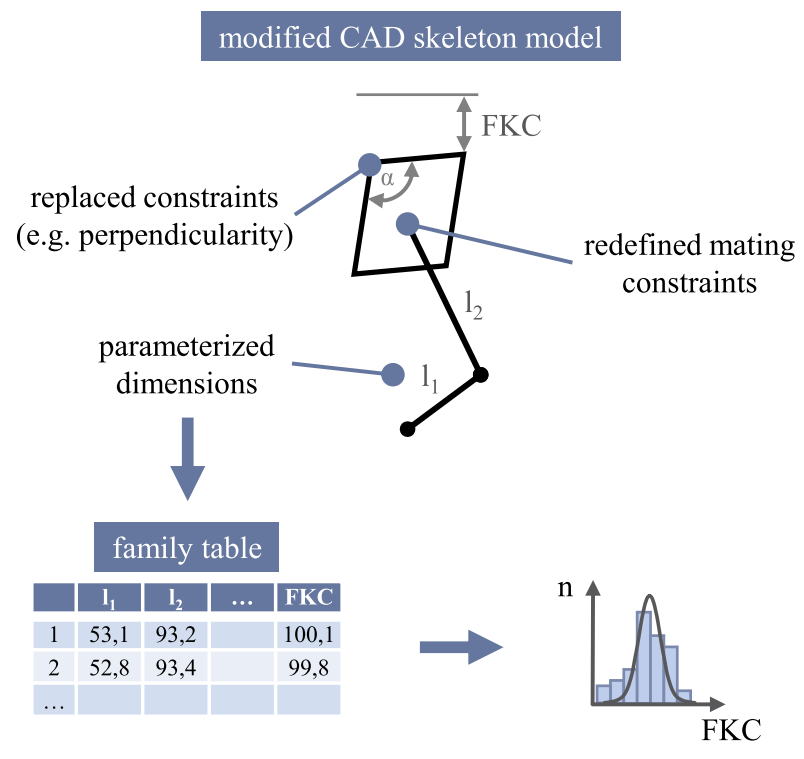

Fig. 8 Simplified workflow of CAD-based tolerance analysis of skeleton models

concept phase and the concrete procedure of the preliminary design.

\subsection{CAD-based tolerance analysis of the preliminary design}

Since the structure of the relevant geometry elements is now already known, the skeleton model representing the preliminary design including the relevant product dimensions is created in the next step of the product development. Thus, a model for the conventional tolerance analysis is available (Ziegler and Wartzack 2013). Although numerous tools have been established for this purpose, the resources are often limited and their acceptance in the design environment is usually low. Since the skeleton model is usually created in the CAD system (e.g., for kinematic analyses) anyway and the product developer is very familiar with it, it is obvious to integrate the tolerance analysis directly into the CAD system (Goetz et al. 2018b). Therefore, the nominal skeleton model is modified to get a fully parametric CAD model that allows a comprehensive mapping of potential variation. As shown in Fig. 8, the design constraints such as perpendicularity are replaced by corresponding dimensions and, if necessary, mating constraints are redefined. Moreover, the influence of geometric tolerances already defined in the tolerance graphs in the previous steps, can be taken into account in an abstract manner by means of a simplified representation of displacements and tilts of adjacent components. The subsequent parameterization of the corresponding dimensions enables the external control of the model via family tables, see Fig. 8. In combination with a proper sampling of the 
varying parameters, this provides detailed information about the system behavior with regard to the analyzed FKC. Since this can be both a parameter variation in the sense of design changes (e.g., the ratio of 11 and 12 in the model in Fig. 8) and in the sense of a tolerance analysis, the method delivers a robust parameter design as well as first estimation of tolerance values. Finally, the CAD-based tolerance analysis can also be used to identify the main contributors to allow an economic adjustment of the tolerance values.

Although the final tolerance analysis based on the detail design cannot be completely replaced, even three-dimensional and over-constrained systems can be reliably examined in accordance with the established vectorial tolerance analysis. The quality of the results depends above all on the level of detail of the model modification and the extent of the variations taken into account. However, it was found that even if the system is limited to the elementary tolerances, reliable statements about the system behavior can be made (Goetz et al. 2018b). Thus, in accordance with Taguchi's parameter and tolerance design (Taguchi et al. 1990), a significant improvement in the robustness of the product can be achieved by gradual adaption of the skeleton model.

At the end of this step, there is a robust product design and a first validated proposal for elementary tolerancing, so that time-consuming iterations in the subsequent detail design (see Fig. 4) are avoided. The proposed methodology thus creates a meaningful link to the detailed tolerance analysis and synthesis at the end of the product development, as exemplarily presented at the end of Sect. 2.2. However, since there are numerous established and user-friendly methods that are well integrated into the late stages of the design process, this is not subject of this contribution.

\section{Application}

In order to demonstrate the benefits and the continuity in a comprehensible way, the methodology is applied to the development of the mechanism of an electric window regulator as an example of industrial relevance. Even though the mechanism under investigation is of low complexity contributing to a good comprehensibility in the context of this contribution, there are alternative principle solutions which are widely used in practice (Zhao 2015) enabling a realistic robustness evaluation. Moreover, the simulation of the kinematic and orientation of the window in the rubber sealing is subject to numerous research projects (Fanghella et al. 2002; Gfrerrer 2011). Since the detailed application of the single methods described in Sects. 3.2-3.5 has already been shown using a scissor lift table and a coinage machine in Goetz et al. (2018a, b, 2019), this section focuses on the continuity and integration into the design process.

\subsection{Prioritization of functional key characteristics}

The product development of the window regulator mechanism starts with the product specification, which is simplified by the central requirement that the window should be opened and closed electrically without jamming. Taking this into account, the function structure partly shown in Fig. 9 is developed in the design process framework. According to the procedure described in Fig. 5, the methodology starts with determining the FKCs corresponding to the functions at this stage. To simplify this step in the context of the contribution, the procedure here is limited to the examination with regard to the movement of the window. This means that in the sense of the QFD, the extent to which individual functions and FKCs contribute to fulfilling the only functional requirement 'non-jamming movement of window' is assessed. Thus, aspects associated with the sealing of the window can be ignored in this case. Although initially both translational and rotational displacements of the windows might be defined as essential FKCs, the QFD-oriented assessment shows that translational variations of the window can be neglected due to the small effect on the functional requirement. Instead, the tilting plays a major role in the movement along the rubber seal, see Fig. 9. Furthermore, abstract dimensions such as the clearance in the mechanism to avoid jamming are assessed as important FKCs. For reasons of clarity, less relevant functions and FKCs such as the translational variations are not shown in Fig. 9, so that it is limited to the characteristics relevant for the further process. In addition, according to the description in Goetz et al. (2019), the remaining functions are weighted equally with 1.

While so far only qualitative prioritization of relevant elements has taken place, the next step quantifies the significance of the individual FKCs. Therefore, the simplified formula Eq. 1 for the VRPN calculation of the VMEA-based approach described in Sect. 3.2 and Goetz et al. (2019) is used. The two coefficients $I$ and $P$ represent the effect and the probability of occurrence of variations and vary from

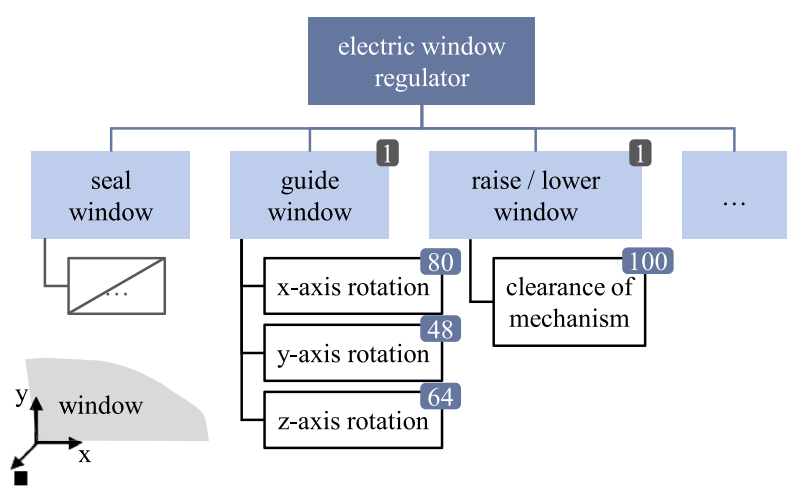

Fig. 9 Extract of function structure including the relevant FKCs (color figure online) 
1 to 10 . The factor $C$ respects the potential of corrective adjustments and as in this case, it should be set to 1 without in-depth expert knowledge about the product.

$V R P N=I * P * C$

with $I$ : impact of $\mathrm{FKC}$ on the product,

$P$ : probability of variation of $\mathrm{FKC}$,

$C$ : adjustment potential.

Since variations in the clearance of the mechanism are likely and can lead to a malfunctioning of the whole system, the evaluation results in a VRPN of 100 for the corresponding FKC. Although the effects of the tilting are similarly high, the VRPN is mainly lower due to the lower probability of variation $(P)$. This is associated with the bearing of the window along the lower edge. While undesired rotations around the $y$-axis can be easily avoided, tilting around the $x$-axis is likely due to the long extension of the free end. These considerations, which are independent of the final design of the window regulator mechanism, lead to the VRPN values in Fig. 9 reflecting the significance of the FKCs with regard to the entire product. Thus, it is clear for this simplified example that the focus in the further design process is to be put on the rotational variations and in particular on the clearance.

\subsection{Robustness evaluation of principle solutions}

The ongoing product development process initially leads to the three alternative principle solutions for the realization of the window regulator shown in Fig. 10. To find the most robust solution and to take this into account in the evaluation within the framework of the design process, the solutions are evaluated with the robustness matrix proposed in Sect. 3.3. Since the drive unit for the different solutions is the same in each case, the evaluation is here limited to the movement of the mechanism.

In the evaluation process explained in Sect. 3.3, the FKCs are first entered into the robustness matrix weighted with the respective VRPN values, which are highlighted in dark blue

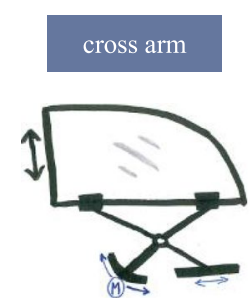

robustness index 16

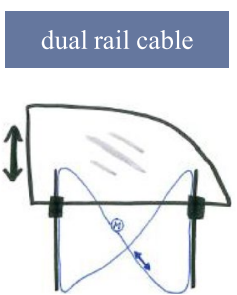

robustness index 9
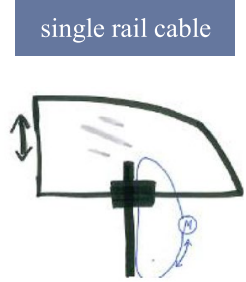

robustness index 10
Fig. 10 Different solutions for an electric window regulator with assigned robustness indices from robustness evaluation matrix in Fig. 9. Subsequently, the criteria (degree of constraint, number of parameters and coupling) proposed in Goetz et al. (2019) will be used for the evaluation of the solutions. For this purpose, the different solutions are compared with each other with regard to the respective criteria and ranked in the matrix. The entire matrix as well as a comprehensive description of the evaluation process presented in the appendix finally leads to the robustness indices shown in Fig. 10. High values indicate a high sensitivity of the solution to variations. The bad result for the cross-arm solution seems plausible, since it contains much more elements or joints and the nearly total decoupling realized by the cable does not apply. Therefore, considering variation, the dual rail cable solution should be preferred for the further product development. However, despite the different robustness, all three solutions have become established in automotive engineering. This shows that the consideration of variations is only one aspect that should be taken into account in the multi-criteria evaluation in the design process.

\subsection{Graph-based tolerance specification and analysis of the product structure}

Assuming that the dual rail cable solution was chosen for the product development, a product structure graph is created in the next step of the proposed method, see left in Fig. 11. Since the solutions in Fig. 10 already represent a detailed structure of the product, this can just be regarded as an alternative form of representation. However, in the case of new developments of complex systems usually consisting of several partial solutions, the structuring of the principle solutions is an intermediate step in the product development process.

The resulting graph in Fig. 11 includes the relevant components corresponding to the focused mechanism of the window regulator. Although the dual rail cable concept is

\section{dual rail cable}

fix - fixed contact | linear - 1D sliding contact | rot - cylindrical joint

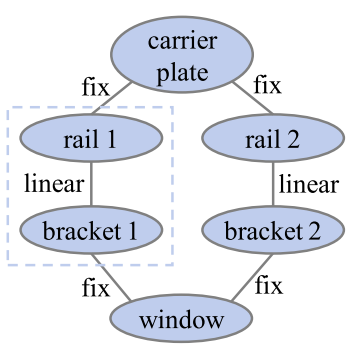

Fig. 11 Comparison of graphs representing the product structure on component level (blue dashed box shows the section, which is shown in detail in Fig. 12) (color figure online) 
already considered as being robust in the previous step, it is compared with the structure of the cross-arm concept. Even though this is not intended in the proposed method, it serves here to show the benefit of the previous step described in Sect. 3.3 and to verify the robustness evaluation. As already mentioned in the previous section, the comparison between the two graphs in Fig. 11 visually reveals that both the larger number of elements and joints as well as the coupling indicate a low robustness of the cross-arm solution. Although this demonstrates the robustness of the selected solution, the graphical representation of the product structure helps to identify remaining weak points in the design. So the two linear contacts, each with one degree of freedom in the same direction (see Fig. 11), indicate a potential jamming in the graph loop. This could be avoided by a tight parallelism tolerance between the two rails, sufficient clearance in the contact area or the use of contacts with additional degrees of freedom. In this way, weak points of the design can be eliminated on the basis of the product structure even before the initial preliminary design.

After a brief excursus revealing the benefits of the graphical approach, the proposed methodology continues with the extension of the product structure graph to the tolerance structure graph. Therefore, the labels of components and interface relations within the product structure graph already imply the involved relevant geometry elements and their corresponding tolerances. Since frequently recurring elements are mainly used in the design environment, the product structure graph can be translated semi-automatically into a tolerance structure graph (Goetz et al. 2018a) as shown in extracts in Fig. 12. For the sake of traceability, it is limited to the contact between rail and bracket, see blue dashed box in Fig. 11. By defining a linear sliding contact between both

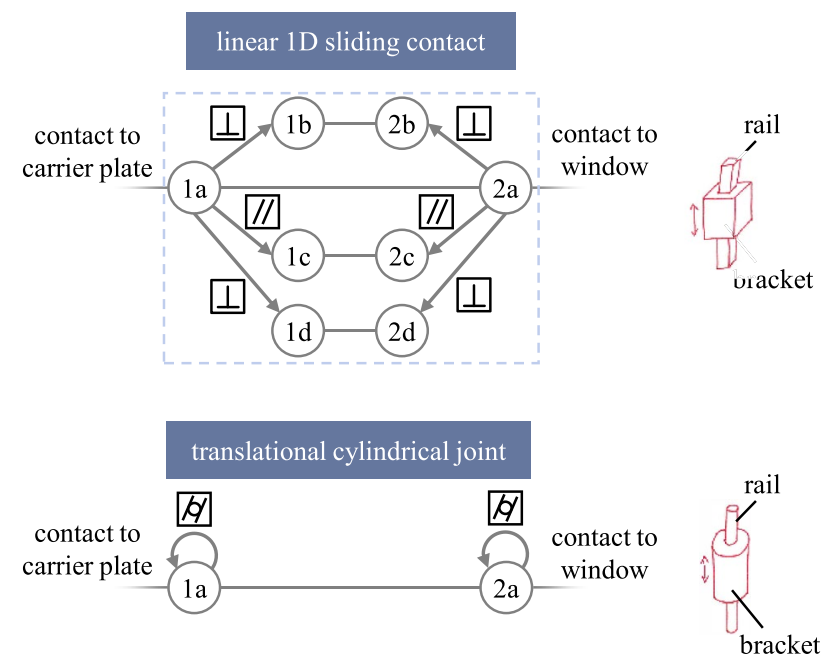

Fig. 12 Extract of tolerance structure graph for the contact between rail and contact of the dual rail cable regulator. The lower section shows an alternative contact concept components, the eight planar faces and the corresponding elementary tolerances are already pre-defined, regardless of the final product design.

To reduce the resulting overall tolerance structure graph with regard to the functional requirements, the previously defined FKCs such as the rotational variation of the window referring to the carrier plate is integrated in the graph. Through an automatic reduction to the corresponding tolerance chains, it becomes clear that the linear contact has a significant influence on the path from carrier plate to window (see Fig. 11) due to the many geometry elements involved. While complex systems first focus on the spatial arrangement, this example concentrates on robust contact definitions. Thus, an alternative translational cylindrical joint with a higher degree of freedom is used instead, so that the tolerance structure graph is reduced to two cylindrical surfaces in contact, see lower part of Fig. 12. This conceptual change is in line with the previous step's demand for a multiple degrees of freedom contact, resulting in a more robust product according to the axiomatic design (Suh 1990). Alternatively, the demand for a higher degree of freedom in contact, revealed by the graphs, can also be realized in the context of product development by considering clearance. Thus, the structural design, the definitions of the essential contacts in the product and potential weak points are available for the designer even before the definition of the essential product dimensions.

\subsection{CAD-based tolerance analysis of the preliminary design}

In particular, the contact definitions played a major role in the creation of a first preliminary design in form of a skeleton model of the dual rail cable regulator. As can be seen in Fig. 13, the skeleton is assembled from local coordinate systems, axes and simple geometries. Referring to the global coordinate system 'ORIGIN', which represents

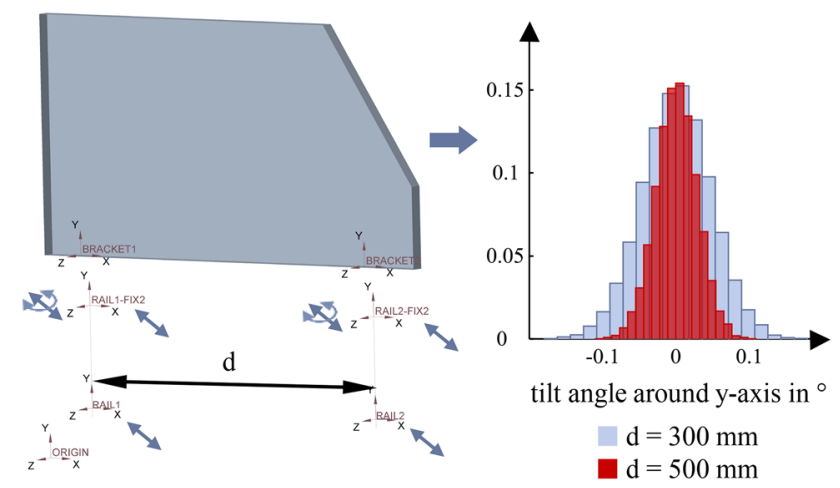

Fig. 13 Skeleton model of dual rail cable regulator with the histogram of the corresponding tilt angle of the window (color figure online) 
the carrier plate, the fixing points for the rails are defined, so that an axis can be defined for the representation of the rail. Moreover, the two brackets are represented with a specific offset from the resulting rail axes. Since the previous step in Sect. 4.3 suggested a translational cylindrical joint at this point, the final rotatory orientation of the brackets results from the assembly of the window. Thus, the window is clearly defined not only in the nominal state but also under variation. The setup of the variational model is done with respect to the qualitative tolerance specification from the tolerance graph and the associated tolerance zones. For a qualitative expedient model, the parameters describing the rotational and translational displacement of the local coordinate systems are varied according to the general tolerances suggested in ISO 2768-1:1989. As the blue arrows in Fig. 13 already indicate, the fixing points for the rails are displaced in three dimensions, while the brackets are additionally rotated. The subsequent tolerance analysis in Creo Parametric $4.0^{\circledR}$ finally leads to among others the distribution for the $y$-axis rotation of the window, which was initially defined as a FKC in Fig. 9. Moreover, the influence of individual design parameters was evaluated. For example, Fig. 13 shows the distributions for the rotation of the window around the vertical $y$-axis for two different distances $d$ between the two rails of the regulator.

As already expected, the histogram on the right confirms the assumption that the system becomes more robust as the distance between the rails increases. Besides the optimization of parameters in the sense of the parameter design (Taguchi et al. 1990), it enables an adaption of the tolerance values, whereby the associated tolerance zones facilitate the transfer to a standard-compliant tolerancing for the subsequent detail design stage. Moreover, a sensitivity analysis identifies the tolerances to which particular attention must be paid in the further design process. In this case study, for example, the positioning of the upper fixing points of the rails is crucial for the resulting rotation around the $y$-axis.

\section{Discussion}

The proposed methodology, thus, represents an approach that allows a thorough consideration of variations, especially in the early product development stages prior to the detail design. In addition to the individual modular approaches assigned to the single phases of the design process, the potential of this methodology lies above all in the continuous utilization of product and tolerance information from the preceding steps, see Fig. 4. However, the proposed methodology covers a vast area of product development, robust design as well as the tolerancing process and initiates a shift of competences. Therefore, its practical usability in industry requires discussion. So, this section critically deals with current limitations and the verification of the approach. Since a reliable quantitative validation of such tolerancing approaches requiring manufacturing information is expensive, the approach has currently been tested using simplified case studies, see first paragraph in Sect. 4. The lack of industrial validation is partly due to the fact that the approach presented is currently primarily a new procedure for the integrated tolerance management in early design stages rather than a fully automated software tool. However, the applicability, reproducibility and reliability of the approach was confirmed by comparative tolerance analyses and an undergraduate student with the aim to reveal current weak points for unexperienced users.

Thus, even though the functional requirements as well as the function structure is already available in the first step of the proposed approach (see Fig. 4), the proper definition and selection of relevant FKCs depends on the designer's experience. However, this challenging process, which is thoroughly described in Zheng et al. (2008), is improved by a further linking of requirements, functions and FKCs. This helps to gain experience knowledge and thus objectifies the process. However, due to the remaining subjective influence, the resulting VRPN values from the subsequent step are subject to uncertainties, so that the weighting shown in Fig. 5 is to be seen as a relative value within a single product and not across products. The hierarchical structure of the approach (Goetz et al. 2019) partially addresses this problem and enables a parallel evaluation of several engineers. Furthermore, although the VRPN values determined in the study with an undergraduate student differ significantly from the values in Fig. 9, the relative weighting of the three rotational FKCs matches with a maximum deviation of $10 \%$.

In a similar way, the robustness index of the approach presented in Sect. 3.3 primarily serves to compare different principle solutions rather than to assign an exact robustness value. However, the structured approach, which considers several robust design aspects, facilitates a reliable comparison of principle solutions. Even though this robustness evaluation is considerably simplified by using the results from the preceding step (Sect. 3.2), the evaluation process is associated with additional effort. However, this evaluation according to the individual criteria already requires a critical discussion of the product in the concept phase and thus accelerates the further product development process in the sense of frontloading (Thomke and Fujimoto 2000). Moreover, the continuous linking of the FKCs from the beginning of product development to production fosters the gain of experience, when using the methodology frequently. Thus, it improves the quality and efficiency of this evaluation.

The subsequent graph-based approach (see Sect. 3.4) is an essential concretization step in the product development process and is mainly facilitated using semantic context. However, in the current state of implementation, this 
requires an accurate predefinition of the contact conditions and components used, so that the benefit is primarily limited to recurring areas of the design. Since, as is known from the decision-making (Weck et al. 2012), similar solutions or designs are predominantly used in product development, this already covers a vast variety of products. Nevertheless, the integration of dimensional tolerances and degree of freedom information is necessary for a fully comprehensive graphbased representation of the product concept and a targeted reduction of the graph. However, the weighting of the FKCs from the first step already contributes to a reliable graph reduction and thus robustness evaluation of the spatial concept arrangement, especially for competing designs.

Although the product developer is supported by the tolerance graph, the current implementation lacks the automatic generation of a skeleton model in the CAD system. Thus, the shortcoming of the method presented in Sect. 3.5 lies in the engineer's decision as to which extent tolerances should be integrated in the model and consequently taken into account in the tolerance analysis. However, it has been shown that CAD-internal visualization allows a straightforward setup of the tolerance analysis model and thus provides quantitative reliable results (Goetz et al. 2018b). For example, the approach can also be used to perform a tolerance analysis of the alternative cross-arm window regulator to compare the robustness of different solutions and thus verify the preceding steps (see Sects. 3.3, 3.4). Using a setup similar to the tolerance analysis model of the dual rail cable regulator described in Sect. 4.4 finally leads to the distribution of the $y$-axis rotation of the window of a cross-arm regulator, see Fig. 14. As the comparison of Figs. 13 and 14 already indicates, the standard deviation of the tilt angle is about five times as high for the cross-arm solution. Even though the distance between the mounting brackets is almost the same in both skeleton models, the cable regulator is more robust. Since this is in line with the robustness evaluation of principle solutions (see Fig. 10), it emphasizes the benefit of the proposed methodology.

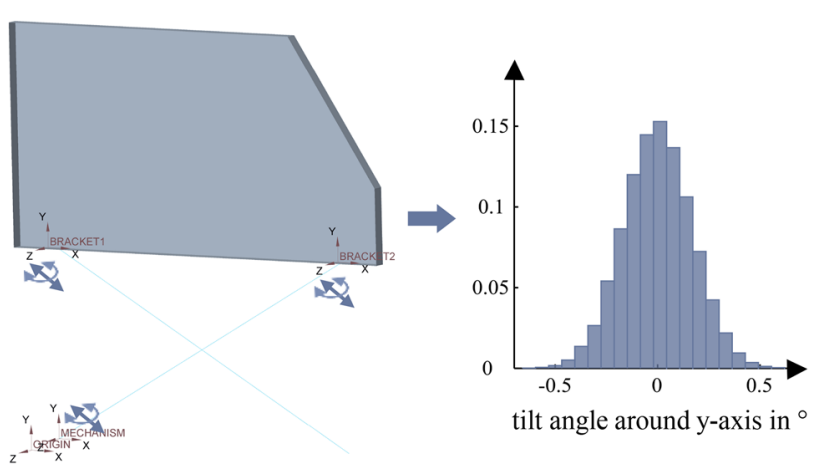

Fig. 14 Skeleton model of cross-arm regulator with the histogram of the corresponding tilt angle of the window
Finally, this points out that the proposed methodology enables the designer to develop robust products as part of the design process leading to a partial shift of tolerancing competences to common design engineers. Thus, the consistent consideration of variations from the beginning of the product development process helps to avoid tight tolerances in the final product or expensive tolerance optimizations.

\section{Summary and outlook}

Based on an extensive literature review, this contribution reveals the lack of straightforward robust design and tolerancing methods applicable in the early stages of the product development process. Moreover, the discrepancy between the two domain perspectives of the design engineer and the tolerance expert leads to the fact that the potential of existing methods taking variation into account remains widely unused in the design environment. As a consequent response to this, this paper presents a holistic methodology in which the design engineer is enabled to develop robust products even before the final product design, so that the scarce resources for the final tolerance design and analysis can be used efficiently. This is mainly achieved by linking information such as FKCs throughout the whole product development process and by closely integrating the approach into the design environment, for example using familiar methods and software. The combination of several newly developed approaches finally leads to a holistic methodology applicable from the beginning of the concept phase to the end of the preliminary design stage.

Based on the function structure, the relevant characteristics are first identified and weighted by a VMEA-based approach with regard to their significance for the entire product. This enables a detailed robustness evaluation of different principle solutions unifying established robustness criteria. Subsequently, the graph-based approach uses semantic context for the proper selection of a robust arrangement of the chosen principle solutions. The associated, semi-automatically concretization closes the gap between the qualitative robustness consideration and the final CAD-based tolerancing approach of the proposed methodology enabling a quantitative tolerance analysis of the preliminary design.

Since the methodology is still characterized by manual steps leading to additional effort in the early stages of the product development, further research concentrates besides the extension of the methods above all on its automation. Therefore, a common database uniting all relevant product and tolerance information (see Fig. 4) of both domains, similar to approaches such as SysML product models, is necessary. This will enable a fully automated transfer of information between the design process and the developed tolerance methods. Moreover, a further formalization of 
relevant design and tolerance knowledge using an ontology would decisively contribute to automating the necessary concretization step between product concept and preliminary design, thus reducing the time needed. Finally, a comprehensive user study will be necessary to quantify the usefulness of the approach. Furthermore, this fosters the identification of weak points and thus helps to improve the usability and acceptance of the proposed methodology.

Acknowledgements Open Access funding provided by Projekt DEAL. The authors thank the German Research Foundation (DFG) for supporting the research project WA 2913/17-1.

Open Access This article is licensed under a Creative Commons Attribution 4.0 International License, which permits use, sharing, adaptation, distribution and reproduction in any medium or format, as long as you give appropriate credit to the original author(s) and the source, provide a link to the Creative Commons licence, and indicate if changes were made. The images or other third party material in this article are included in the article's Creative Commons licence, unless indicated otherwise in a credit line to the material. If material is not included in the article's Creative Commons licence and your intended use is not permitted by statutory regulation or exceeds the permitted use, you will need to obtain permission directly from the copyright holder. To view a copy of this licence, visit http://creativecommons.org/licenses/by/4.0/.

\section{Appendix: Robustness evaluation matrix}

In the following, the robustness evaluation (see Sect. 4) is described in more detail, whereas Fig. 15 represents the determination of the indices shown in Fig. 10. For a holistic view and description of the basic principles, please refer to Goetz et al. (2019). The FKCs including the corresponding VRPN values and the functions from Fig. 9 as well as the robust design criteria with associated weightings proposed in Goetz et al. (2019) are united in the robustness matrix. For the subsequent evaluation of the different principle solutions in the center of the matrix, a simple ranking $(1=\operatorname{good} / 3=\mathrm{bad})$ is used.

Starting with the evaluation of potential static indeterminacy regarding the rotation of the window around the $x$-axis (see Fig. 9), all solutions are evaluated with a 1 , since the interface between window and mechanism is identical for all three solutions. On the other hand, the rotation around $y$-axis and $z$-axis of the single rail solution is due to the single clip less susceptible to potential indeterminacy compared to the other solutions, see Fig. 10. Finally, it is assumed that indeterminacy in the mechanism is more critical for the cross-arm solution due to its architecture.

Furthermore, the rotation around the $x$-axis of the crossarm regulator has a higher number of influencing parameters due to the necessary translational horizontal contact between mechanism and window. In combination with the twofold mounting of the window, this also leads to the evaluation of the FKCs 'rot y' and 'clearance'. On the contrary, since the single clip of the single rail solution requires a full constraint on the rotation of the window around the $z$-axis, there are numerous contributing parameters.

Finally, the coupling of the relevant functions 'guide window' and 'raise/lower window' (see Fig. 9) is evaluated. While the dual rail solution represents a complete decoupling, the coupling in the single rail regulator is slightly stronger due to the single rail and is maximized with the mechanism of the cross-arm window lifter. The subsequent calculation of the individual evaluation values including their weightings leads to the robustness indices shown in Fig. 15.

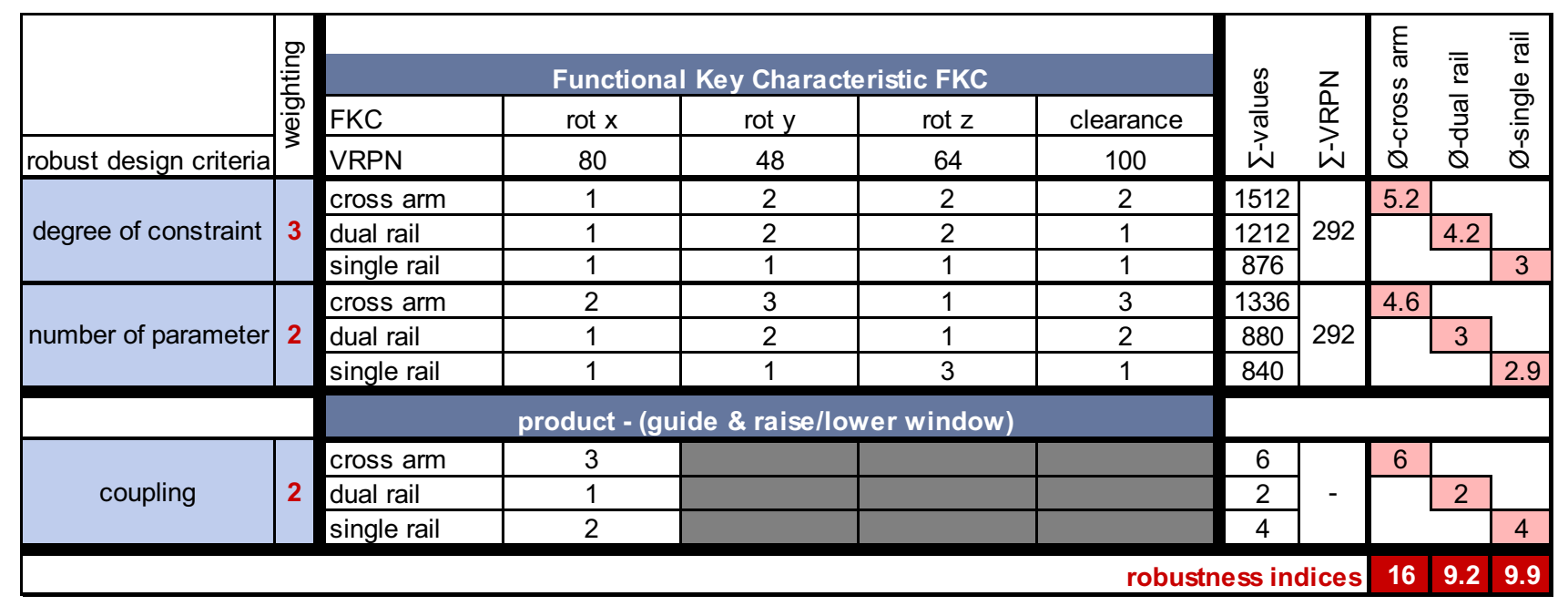

Fig. 15 Robustness evaluation matrix for different principle solutions of a window regulator 


\section{References}

Ameta G, Serge S, Giordano M (2011) Comparison of spatial math models for tolerance analysis: tolerance-maps, deviation domain, and TTRS. J Comput Inf Sci Eng 11(2):021004. https://doi. org/10.1115/1.3593413

Andersson P (1997) On robust design in the conceptual design phase: a qualitative approach. J Eng Des 8(1):75-89. https://doi. org/10.1080/09544829708907953

Anselmetti B (2006) Generation of functional tolerancing based on positioning feature. Comput Aided Des 38(8):902-919. https:// doi.org/10.1016/j.cad.2006.05.005

Armillotta A (2013) A method for computer-aided specification of geometric tolerances. Comput Aided Des 45(12):1604-1616. https://doi.org/10.1016/j.cad.2013.08.007

Ballu A, Mathieu L (1999) Choice of functional specifications using graphs within the framework of education. In: Houten F, Kals $\mathrm{H}$ (eds) Global consistency of tolerances. Springer, Dordrecht, pp 197-206

Ballu A, Falgarone H, Chevassus N, Mathieu L (2006) A new design method based on functions and tolerance specifications for product modelling. CIRP Ann 55(1):139-142. https://doi. org/10.1016/S0007-8506(07)60384-9

Bjarklev K, Eifler T, Mortensen N, Linnebjerg S, Ebro M (2018) Application of a graphical scheme for representing the mode of action of products for identification of key characteristics. Procedia CIRP 75:39-44. https://doi.org/10.1016/j.proci r.2018.04.056

Blessing L, Chakrabarti A (2009) DRM, a design research methodology. Springer, London

Bradley H, Maropoulosa P (1998) A relation-based product model for computer supported early design assessment. J Mater Process Technol 76:88-95. https://doi.org/10.1016/S0924-0136(97)00328-2

Chakhunashvili A, Johansson P, Bergman B (2004) Variation mode and effect analysis. RAMS. https://doi.org/10.1109/ RAMS.2004.1285476

Chan L, Wu M (2002) Quality function deployment: a literature review. Eur J Oper Res 143(3):463-497. https://doi.org/10.1016/S0377 -2217(02)00178-9

Clarkson J, Simons C, Eckert C (2004) Predicting change propagation in complex design. J Mech Des 126(5):788-797. https://doi. org/10.1115/1.1765117

Colosimo B, Senin N (2011) Geometric tolerances. Springer, London

Dantan J, Qureshi A (2009) Worst-case and statistical tolerance analysis based on quantified constraint satisfaction problems and monte carlo simulation. Comput Aided Des 41(1):1-12. https://doi. org/10.1016/j.cad.2008.11.003

Dantan J, Anwer N, Mathieu L (2003) Integrated tolerancing process for conceptual design. CIRP Ann 52(1):135-138. https://doi. org/10.1016/S0007-8506(07)60549-6

Ebro M, Howard T (2016) Robust design principles for reducing variation in functional performance. J Eng Des 27(1):75-92. https:// doi.org/10.1080/09544828.2015.1103844

Ebro M, Howard T, Rasmussen J (2012) The foundation for robust design: enabling robustness through kinematic design and design clarity. Design 2012

Eifler T, Howard T (2018) The importance of robust design methodology: case study of the infamous GM ignition switch recall. Res Eng Des 26(1):39-53. https://doi.org/10.1007/s00163-017-0251-x

Eifler T, Ebro M, Howard T (2013) A classification of the industrial relevance of robust design methods. ICED 2013

Fanghella P, Galliani G, Mezzadra M (2002) Computational recognition of functional parameters of surfaces for the kinematic design of window regulator systems. ASME IDETC CIE. https://doi. org/10.1115/DETC2002/DAC-34060
Ford R (1996) Process for the conceptual design of robust mechanical systems: going beyond parameter design to achieve world-class quality. Stanford University, Stanford

Gfrerrer A, Lang J, Harrich A, Mirz M, Mayr J (2011) Car side window kinematics. Comput Aided Des 43(4):410-416. https://doi. org/10.1016/j.cad.2011.01.009

Giordano M, Pairel E, Hernandez P (2007) Complex mechanical structure tolerancing by means of hyper-graphs. In: Davidson J (ed) Models for computer aided tolerancing in design and manufacturing. Springer, Dordrecht, pp 105-114

Goetz S, Schleich B, Wartzack S (2018a) A new approach to first tolerance evaluations in the conceptual design stage based on tolerance graphs. Procedia CIRP 75:167-172. https://doi.org/10.1016/j. procir.2018.04.030

Goetz S, Schleich B, Wartzack S (2018b) CAD-based tolerance analysis in preliminary design stages enabling early tolerance evaluation. IMECE. https://doi.org/10.1115/IMECE2018-86396

Goetz S, Hartung J, Schleich B, Wartzack S (2019) Robustness evaluation of product concepts based on function structures. ICED. https ://doi.org/10.1017/dsi.2019.359

Göhler S, Howard T (2015) The contradiction index (CI): a new metric combining system complexity and robustness for early design stages. DETC. https://doi.org/10.1115/DETC2015-47255

Göhler S, Husung S, Howard T (2016) The translation between functional requirements and design parameters for robust design. Procedia CIRP 43:106-111. https://doi.org/10.1016/j.proci r.2016.02.028

Göhler S, Ebro M, Howard T (2017) Mechanisms and coherences of robust design methodology: a robust design process proposal. Total Qual Manag Bus 29:239-259. https://doi.org/10.1080/14783 363.2016.1180952

Gremyr I, Hasenkamp T (2011) Practices of robust design methodology in practice. TQM J 23(1):47-58. https://doi.org/10.1108/17542 731111097489

Hasenkamp T, Arvidsson M, Gremyr I (2009) A review of practices for robust design methodology. J Eng Des 20(6):645-657. https ://doi.org/10.1080/09544820802275557

Howard T, Eifler T, Pedersen S, Göhler S, Boorla S, Christensen M (2017) The variation management framework (VMF): a unifying graphical representation of robust design. Qual Eng 29(4):563572. https://doi.org/10.1080/08982112.2016.1272121

Hsu W, Woon I (1998) Current research in the conceptual design of mechanical products. Comput Aided Des 30(5):377-389. https:// doi.org/10.1016/S0010-4485(97)00101-2

Islam M (2009) A dimensioning and tolerancing methodology for concurrent engineering applications II: comprehensive solution strategy. Int J Adv Manuf Technol 42:922-939. https://doi. org/10.1007/s00170-008-1707-y

Johannesson H, Söderberg R (2000) Structure and matrix models for tolerance analysis from configuration to detail design. Res Eng Des 12(2):112-125. https://doi.org/10.1007/s001630050027

Jugulum R, Frey D (2007) Toward a taxonomy of concept designs for improved robustness. J Eng Des 18(2):139-156. https://doi. org/10.1080/09544820600731496

Kremer G (2008) Concept selection methods-a literature review from 1980 to 2008. Int J Des Eng 1(3):243-277. https://doi. org/10.1504/IJDE.2008.023764

Ledoux Y, Teissandier D (2013) Tolerance analysis of a product coupling geometric and architectural specifications in a probabilistic approach. Res Eng Des 24(3):297-311. https://doi.org/10.1007/ s00163-012-0146-9

Lough K, Stone R, Tumer I (2009) The risk in early design method. J Eng Des 20(2):155-173. https://doi.org/10.1080/0954482070 1684271

Mathias J, Kloberdanz H, Eifler T, Engelhardt R, Wiebel M, Birkhofer H, Bohn A (2011) Selection of physical effects based on 
disturbances and robustness ratios in the early phases of robust design. ICED 11(5):324-335

Mathieu L, Marguet B (2001) Integrated design method to improve producibility based on product key characteristics and assembly sequences. CIRP Ann 50(1):85-88. https://doi.org/10.1016/S0007 -8506(07)62077-0

Matthiassen B (1997) Design for Robustness and Reliability. Dissertation, Technical University of Denmark

Myszka D (2012) Machines and mechanisms: applied kinematic analysis. Pearson Education, Upper Saddle River

Pahl G, Beitz W, Blessing L, Feldhusen J, Grote K, Wallace K (2007) Engineering design: a systematic approach, 3rd edn. Springer, London

Polini W (2012) Taxonomy of models for tolerance analysis in assembling. Int J Prod Res 50(7):2014-2029. https://doi. org/10.1080/00207543.2011.576275

Polini W (2016) Concurrent tolerance design. Res Eng Des 27(1):2326. https://doi.org/10.1007/s00163-015-0203-2

Pugh S (1981) Concept selection, a method that works. ICED 81

Qin Y, Qi Q, Lu W, Liu S, Scott P, Jiang X (2017) A review of representation models of tolerance information. Int J Adv Manuf Technol 95:2193-2206. https://doi.org/10.1007/s00170-017-1352-4

Reich Y (2008) Preventing breakthroughs from breakdowns. ESDA. https://doi.org/10.1115/ESDA2008-59189

Reich Y, Subrahmanian E (2015) Designing PSI: an introduction to the PSI framework. ICED 15

Roy U, Pramanik N, Sudarsan R, Sriram R, Lyons K (2001) Function-to-form mapping: model, representation and applications in design synthesis. Comput Aided Des 33(10):699-719. https://doi. org/10.1016/S0010-4485(00)00100-7

Shah J, Ameta G, Shen Z, Davidson J (2007) Navigating the tolerance analysis maze. Comput Aided Des Appl 4(5):705-718. https://doi. org/10.1080/16864360.2007.10738504

Shen Z, Shah J, Davidson J (2008) Analysis neutral data structure for gd\&t. J Intell Manuf 19(4):455-472. https://doi.org/10.1007/ s10845-008-0096-2

Söderberg R, Lindkvist L (1999) Computer aided assembly robustness evaluation. J Eng Des 10(2):165-181. https://doi. org/10.1080/095448299261371

Söderberg R, Lindkvist L, Wärmefjord K, Carlson J (2016) Virtual geometry assurance process and toolbox. Procedia CIRP 43:3-12. https://doi.org/10.1016/j.procir.2016.02.043

Srinivasan R, Wood K, McAdams D (1996) Functional tolerancing: a design for manufacturing methodology. Res Eng Des 8(2):99-115. https://doi.org/10.1007/BF01607864

Suh N (1990) The principles of design. Oxford University Press, New York
Suh N (1998) Axiomatic design theory for systems. Res Eng Des 10(4):189-209. https://doi.org/10.1007/s001639870001

Sullivan L (1986) Quality function deployment. Qual Prog 19(6):39-50

Taguchi G, Elsayed E, Hsiang T (1990) Quality engineering in production systems. McGraw Hill, New York

Taguchi G, Yano H, Chowdhury S, Taguchi S (2005) Taguchi’s quality engineering handbook. Wiley, Hoboken

Thomke S, Fujimoto T (2000) The effect of "front-loading" problemsolving on product development performance. J Prod Innov Manag 17(2):128-142. https://doi.org/10.1016/S0737-6782(99)00031-4

Thornton A (1999) Variation risk management using modeling and simulation. J Mech Des 121(2):297-304. https://doi. org/10.1115/1.2829457

Thornton A (2004) Variation risk management: focusing quality improvements in product development and production. Wiley, Hoboken

Ullmann D (2009) The mechanical design process. McGraw Hill, New York

Ulrich K, Pearson, S (1993) Does product design really determine 80\% of manufacturing cost? Working Paper WP\#3601-93. Alfred P. Sloan School of Management, MIT, Cambridge

Weber C, Werner H, Deubel T (2003) A different view on product data management/product life-cycle management and its future potentials. J Eng Des 14(4):447-464. https://doi.org/10.1080/09544 820310001606876

Weck O, Eckert C, Clarkson J (2012) Guest editorial. Res Eng Des 23(4):265-267. https://doi.org/10.1007/s00163-012-0143-Z

Zawiślak S, Rysiński J (2017) Graph-based modelling in engineering. Springer, Cham

Zhao X (2015) A Contribution to the Computer Aided Development of an Electrical Window Regulator System. Dissertation, TU Ilmenau

Zheng L, McMahon C, Li L, Ding L, Jamshidi J (2008) Key characteristics management in product lifecycle management: a survey of methodologies and practices. J Eng Manuf 222(8):989-1008. https://doi.org/10.1243/09544054JEM1045

Ziegler P, Wartzack S (2013) Concept for tolerance design in early design stages based on skeleton models. ICED 13(5):1-10

Publisher's Note Springer Nature remains neutral with regard to jurisdictional claims in published maps and institutional affiliations. 